\title{
Early diagnosis of celiac disease in IgA deficient children: contribution of a point-of-care test
}

\author{
Francoise Bienvenu', Silvia I Anghel ${ }^{2 *}$, Cécile Besson Duvanel ${ }^{2}$, Julien Guillemaud ${ }^{1}$, Lorna Garnier ${ }^{1,3}$, Florian Renosi $^{1,3}$,
} Alain Lachaux ${ }^{3}$ and Jacques Bienvenu ${ }^{1}$

\begin{abstract}
Background: The serological diagnosis of celiac disease (CD) often relies on the presence of anti-tissue transglutaminase (tTG) IgA autoantibodies. Patients suffering from selective IgA deficiency (IgAD) are often not aware of their IgA deficiency and are tested as CD negative, delaying considerably the diagnosis. The detection of IgG against deamidated gliadin peptides (DGP) has high specificity and better sensitivity than IgG anti-tTG. A multi-analytic lateral-flow immunochromatographic assay (CD-LFIA) based on the detection of IgA and IgG anti-DGP and total IgA was shown to have a good diagnostic accuracy for CD. The aim of this study was to evaluate the clinical accuracy of its use in children suffering from IgAD.

Methods: 45 IgAD children ranging from 1.1 to 17.4 years and suspected of CD or having high CD risk factors were referred from outpatient clinics located in the area of Rhone-Alpes (France) to the Hospices Civils de Lyon, Paediatric Hospital-Gastroenterology-Hepatology- Nutrition Department for further CD investigations. The CD investigations, including the sample collection, were performed within the Paediatric Hospital-GastroenterologyHepatology- Nutrition Department, and the serological testing was performed at the Lyon-Sud Hospital-Immunology Laboratory. The diagnosis of CD was based on IgG anti-tTG serology, biopsy results and patient follow-up. The serum samples were retrospectively tested on the CD-LFIA test.
\end{abstract}

Results: A total of eight (8) patients were diagnosed as new CD. All were correctly identified by the CD-LFIA. The test yielded four (4) false positive results. Two patients with positive IgG anti-tTG were negative on CD-LFIA, but were classified as CD negative based on biopsy results and patient follow-up. The remaining 33 patients were found negative by both methods. The specificity and sensitivity of CD-LFIA was of 89.2\% [74.6-97.0] and of 100\% [63.1-100] respectively. The negative predictive value (NPV) was of 100\% [89.4-100], and the Likelihood Ratio for Negative Test (LR-) was of 0 [0.0-0.91].

Conclusions: CD-LFIA is a useful, non-invasive and rapid tool to rule out CD in primary care paediatric patients having CD-related symptoms and IgAD. Patients having a positive CD-LFIA result could be then readily directed to secondary care setting for further evaluation by standard serology and biopsy.

Keywords: Celiac disease, IgA deficiency, Deamidated gliadin peptides, Point-of-care test

\section{Background}

Selective IgA deficiency (IgAD) is a common immunodeficiency occurring in Caucasians with a prevalence rising up to 1:600 [1,2]. IgAD is characterized by total IgA serum levels below $0.06 \mathrm{~g} / \mathrm{L}$ and normal levels of IgM and IgG [3]. Although the majority of IgAD individuals are asymptomatic, IgAD is associated with autoimmune

\footnotetext{
* Correspondence: silvia.anghel@augurix.com

${ }^{2}$ Augurix SA, Monthey $\mathrm{CH}-1870$, Switzerland

Full list of author information is available at the end of the article
}

disorders such as celiac disease (CD) [4]. Its frequency is raised and estimated to be about 1:40 among patients suffering from $C D$ [5-7].

The European Society for Paediatric Gastroenterology Hepatology and Nutrition (ESPGHAN) strongly recommends testing for the presence of IgA autoantibodies against tissue transglutaminase (tTG) as the initial step for $\mathrm{CD}$ diagnosis $[5,8,9]$. Moreover, in cases where the total IgA status is unknown, the guidelines strongly recommend IgA measurement [8-10]. In case of IgAD, a positive 
IgG anti-Endomysium (EMA) [8], anti- tTG or anti- deamidated gliadin peptides (DGP) antibodies is considered as diagnostically relevant [8-10].

Unfortunately, investigation of the total IgA levels is frequently neglected, and patients suffering from IgAD are often not aware of their IgA deficiency and may therefore be tested as $\mathrm{CD}$ negative, delaying considerably the diagnosis $[3,11]$. Recently, it has been demonstrated that the detection of IgG against DGP has a high specificity and a better sensitivity than IgG anti-tTG [12-16]. Therefore, their use was suggested as an alternative for the diagnosis of CD in IgAD patients [3].

A multi-analytic lateral-flow immunochromatographic assay (CD-LFIA) based on the rapid detection of both IgA and IgG anti-DGP and total IgA has previously been shown to have a good diagnostic accuracy to rule out $\mathrm{CD}$ in high-risk paediatric and adult populations $[17,18]$.

The aim of the present study was to evaluate its use as a tool to rule out CD in children suffering from IgAD.

\section{Methods \\ Patients}

Paediatric patients suspected of CD or having high CD risk factors were referred from outpatient clinics located in the area of Rhone-Alpes (France) to the Hospices Civils de Lyon, Paediatric Hospital-GastroenterologyHepatology- Nutrition Department for further CD investigations. The $C D$ investigations, including the sample collection, were performed within the Paediatric HospitalGastroenterology-Hepatology- Nutrition Department, and the serological testing was performed at the Lyon-Sud Hospital-Immunology Laboratory. From 2001 to 2012, 45 paediatric patients were selectively diagnosed with IgAD with total IgA levels below $0.06 \mathrm{~g} / \mathrm{L}$. The study stems from collaboration between the Paediatric HospitalGastroenterology-Hepatology- Nutrition Department and the Lyon-Sud Hospital-Immunology Laboratory. Ethical approval was obtained from the Ethical Committee of Hospices Civils de Lyon for the retrospective use of the samples collected within the Paediatric HospitalGastroenterology-Hepatology- Nutrition Department and analyzed within the Lyon-Sud Hospital-Immunology Laboratory. The samples were anonymized, and information was collected on the CD related symptoms and/or high risk factors, on the serological investigation, biopsy results, and finally on the patient follow-up when available. From the 45 IgAD paediatric patients, 17 were tested because of clinical suspicion of CD (diarrhea, anemia, and failure to thrive), 18 because of risk factors for CD (autoimmune diseases among which 14 suffer from type 1 diabetes), and finally ten (10) patients were not clinically documented. To our knowledge, none of the patients included in this study were diagnosed with another small intestinal disorder at the time of CD diagnosis.

\section{Diagnostic methods}

Total IgA was measured using the BNII nephelometer (Siemens) according to the manufacturer's protocol. IgAD was diagnosed when total IgA levels were below $0.06 \mathrm{~g} / \mathrm{L}$. For the study population, normal values ranged between $0.12 \mathrm{~g} / \mathrm{L}$ and $2.03 \mathrm{~g} / \mathrm{L}$, depending on the patient's age.

The CD diagnosis was made by the gastroenterologist, and was based on results of serologic enzyme-linked immunosorbent assay (ELISA) IgG anti-tTG test (see below), small intestine mucosal biopsy examination and on patient follow-up when available.

IgG anti-tTG levels were measured by the ELISA test Celikey $^{\oplus}$ from Thermo Fisher (Uppsala, Sweden). Concentrations $>5 \mathrm{U} / \mathrm{mL}$ were considered as positive.

In case of discrepancy between IgG anti-tTG and CDLFIA, IgG anti-DGP levels were measured by the ELISA test Varelisa ${ }^{\bullet}$ Gliadin Antibodies from Thermo Fisher (Uppsala, Sweden). Concentrations $>10 \mathrm{U} / \mathrm{mL}$ were considered as positive.

At endoscopy, 4-6 duodenal biopsies were taken from patients having a positive IgG anti-tTG serology [8]. The duodenal biopsies were analyzed by an experienced histopathologist, who assessed the following pathologic features of CD: villous atrophy, crypt hyperplasia, increased intraepithelial lymphocytes, and chronic inflammation in the lamina propria. The diagnosis of $\mathrm{CD}$ was subsequently confirmed according to the modified Oberhuber-Marsh classification [19].

\section{CD-LFIA test}

The serum samples were tested by CD-LFIA test (Simtomax ${ }^{\odot}$ Augurix, Switzerland) according to the manufacturer's instructions. The CD-LFIA point-of-care device detects simultaneously both human IgA and IgG antiDGP, as well as total IgA in 10 to 15 minutes $[17,18]$. Briefly, the test was read as CD positive and IgAD when both the control line and the line corresponding to antiDGP detection could be seen. Each sample was tested on one device by two independent user-operators blinded to the subject's history and laboratory findings. Prior to the clinical study, each user-operator had completed a oneday course on the use of CD-LFIA.

\section{Statistical analysis}

The laboratory ELISA IgG anti-tTG, biopsy results, and patient follow-up were used as "standard diagnosis" for comparative analyses to evaluate the testing features of CD-LFIA.

The CD-LFIA test's sensitivity, specificity, diagnostic accuracy, and positive and negative likelihood ratios (LR+, LR-) and their corresponding 95\% confidence intervals (CIs) were calculated using the STATA software (version 11; College Station, TX, USA). 


\section{Results}

Patient's characteristics

The diagnostic performance of CD-LFIA was retrospectively tested on serum samples from 45 paediatric patients diagnosed as IgAD, and initially tested by ELISA and biopsy (when requested) because of clinical suspicion of CD or risk factors for CD. Ten (10) patients were not clinically documented.

The age of the population ranged from 1.1 to 17.4 years, with a mean and median age of 8.2 and 8.4 years, respectively, with $40 \%$ of females $(n=18)$, and $60 \%$ of males $(n=27) .50 \%$ of females were initially investigated because of CD-related symptoms, whereas $28 \%$ of them were at high-risk of CD (diagnosed with autoimmune diseases). In contrast, the majority of the male population was suffering from autoimmune disease (48\%, with a clear overrepresentation of type 1 diabetes, $n=11$ ), whereas $30 \%$ were suffering initially from CD-related symptoms (Table 1).

\section{Overall agreement between CD-LFIA and the "standard diagnosis"}

Based on the "standard diagnosis" (IgG anti-tTG positive serology, biopsy result and patient follow-up), eight (8) new cases of $C D$ were found, all were correctly identified by CD-LFIA (Figure 1). Their histology revealed subtotal $(\mathrm{n}=1$; Marsh $3 \mathrm{~b})$ or complete villous atrophy $(\mathrm{n}=7$; Marsh 3c).

From these eight cases, five (5) were among females and were all initially tested because of CD related symptoms, particularly diarrhea, whereas among the three (3) newly identified celiac positive male patients, one had CD related symptoms, and two (2) were suffering from type 1 diabetes. The age of the newly diagnosed CD ranged from 1.5 to 17.4 years.

Thirty-seven patients were diagnosed as celiac negative. Among them, 33 were correctly identified by CD-LFIA. Four (4) patients were identified as positive on CD-LFIA. The IgG anti-DGP levels of these four (4) patients were further measured by ELISA and among them, two (2) had positive levels of IgG anti-DGP. One of these patients underwent duodenal biopsy one year later, and the result showed a negative histology, and was therefore considered as $C D$ negative. The second patient (2.1 years old), showed IgG anti-tTG levels near the cut-off $(3.4 \mathrm{U} / \mathrm{ml}$ instead of $5 \mathrm{U} / \mathrm{ml}$ ) and positive levels of IgG anti-DGP (16 U/ml). Unfortunately, this patient had no follow-up and no biopsy was conducted. Both patients were further considered as false positive (Figure 1).

Among the celiac negative patients, two (2) patients were weakly positive on the ELISA IgG anti-tTG (5 and 9.9 $\mathrm{U} / \mathrm{ml}$ ) and negative on CD-LFIA and ELISA IgG anti-DGP. Based on their medical follow-up and their normal biopsies they were considered as CD negative. Thus, no false negative results were detected using CDLFIA (Figure 1).

\section{Diagnosis in patients suffering from other autoimmune disease}

Among the 45 paediatric patients, 18 were suffering of other autoimmune diseases, among which 15 were suffering of type 1 diabetes. Fourteen (14) of them had concordant results between ELISA IgG anti-tTG, ELISA IgG anti-DGP and CD-LFIA.

Among the four remaining patients, two patients ( 6 and 15.6 years males) had positive ELISA IgG anti-tTG results (5 and $9.9 \mathrm{U} / \mathrm{ml}$ ) but were diagnosed as CD negative based on their biopsy and follow-up. Both patients had negative CD-LFIA and ELISA IgG anti-DGP results. A 5.6 years old male suffering from type 1 diabetes was found negative on both ELISA IgG anti-tTG and anti-DGP but was positive on CD-LFIA. Finally, an 11.2 years old female diagnosed as CD negative based on ELISA IgG anti-tTG result had a negative CD-LFIA result and a positive ELISA IgG anti-DGP result (43 U/ml). Unfortunately, no biopsy and further follow-up was conducted.

\section{Evaluation of the diagnostic performance of CD-LFIA}

These results yield a sensitivity for CD-LFIA device of $100 \%$ [63.1-100], and a specificity of $89.2 \%$ [74.6-97.0] (Figure 2). The Negative Predictive Value (NPV), which takes into account false negative results, was of $100 \%$ [89.4-100] and the Likelihood Ratio for Negative Test (LR-) was of 0 [0.0-0.91] (Figure 2). The overall diagnostic accuracy was of $91.1 \%$ [78.8-97.5].

\section{Discussion}

The diagnosis of CD is a challenge for many physicians. Unfortunately, up to $75 \%$ of CD remains undiagnosed, and the time between the onset of symptoms and diagnosis is often too long [20,21]. It is thought that the use of a highly sensitive rapid point- of- care test, performed in primary care centers, may help physicians to rule out CD and to select patients on which more invasive diagnostic

Table 1 Characteristics of patients participating to the study

\begin{tabular}{|c|c|c|c|c|c|c|c|}
\hline Gender & $\begin{array}{c}\text { Total } \\
\text { population }\end{array}$ & $\begin{array}{l}\text { Population of CD } \\
\text { related symptoms }\end{array}$ & $\begin{array}{l}\text { Population having risk factors } \\
\text { (Autoimmune disease) }\end{array}$ & $\begin{array}{c}\text { Population having } \\
\text { unknown clinical symptoms }\end{array}$ & $\begin{array}{l}\text { Age range } \\
\text { (years) }\end{array}$ & $\begin{array}{l}\text { Mean age } \\
\text { (years) }\end{array}$ & $\begin{array}{l}\text { Median age } \\
\text { (years) }\end{array}$ \\
\hline Females & 18 & 9 & 5 & 4 & 1.3 to 17.4 & 8.4 & 8.8 \\
\hline Males & 27 & 8 & 13 & 6 & 1.1 to 16.2 & 8.1 & 7.3 \\
\hline
\end{tabular}




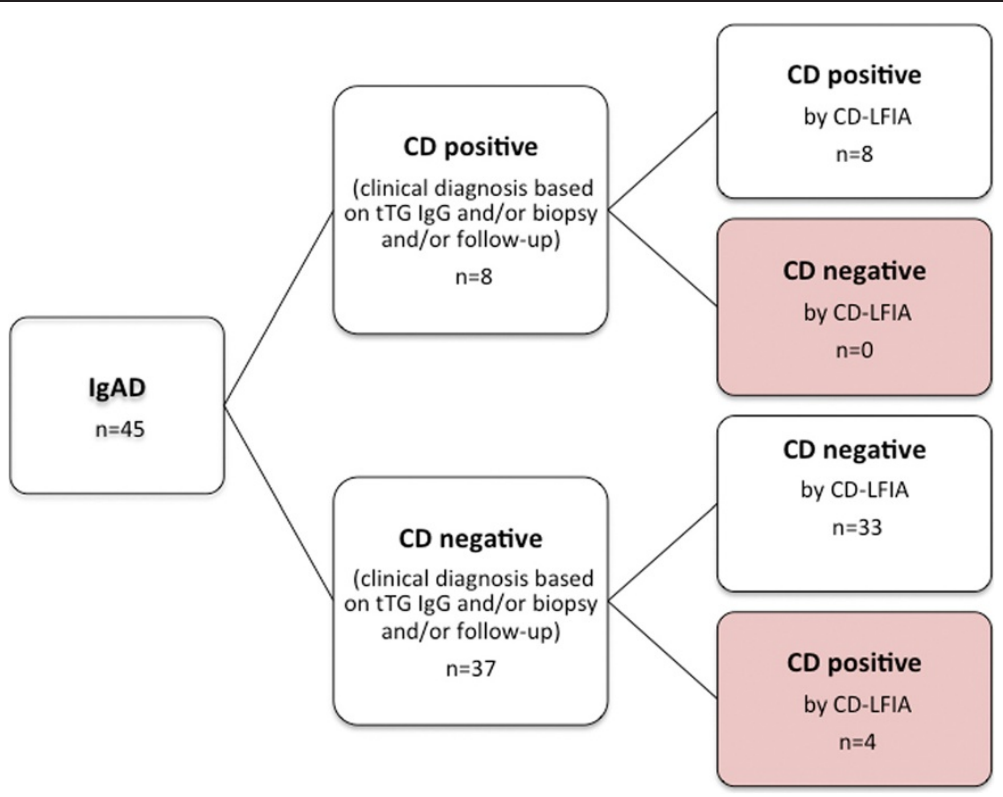

Figure 1 Histogram comparing standard diagnosis by IgG anti-tTG ELISA assay and/or biopsy and/or follow-up to CD-LFIA test results.

tests are required [22-24]. This strategy could allow a better and more reactive patient support, decreasing the time to diagnosis while increasing the patient quality of life.

According to the last ESPGHAN guidelines, the initial testing of patients suspected of CD should be the serological identification of IgA autoantibodies against tTG, followed by biopsy in case of a positive serology [8]. In case of serology/biopsy disagreement, measurement of total IgA levels and IgG anti-tTG or anti-DGP as well as HLA-DQ genotyping are recommended. However, based on epidemiological studies, it is known that IgAD occurs with a higher frequency in CD positive patients [2,4-7]. Regardless the fact that ESPGHAN recommends the measurement of total IgA levels [8] their determination is often neglected, which obviously delays even more the time to diagnosis of $\mathrm{CD}$ in patients suffering from IgA deficiency. Therefore, there is a clear need for reliable and easy to perform tests in primary care centers, that could detect $C D$ regardless of the total IgA status of patient.

As mentioned above, the initial standard serological test is based on IgA anti-tTG antibodies. In case of IgAD, one can think of using IgG anti-tTG or anti-EMA antibodies. However, several clinical studies showed that IgG anti-tTG antibodies are not as sensitive and specific as the IgA anti-tTG antibodies, and the use of EMA assay in daily practice is limited by high costs and subjective interpretations [25]. In contrast, several studies

\section{Celiac Disease}

\begin{tabular}{|l|l|c|c|c|}
\cline { 3 - 4 } \multicolumn{2}{c|}{} & \multicolumn{3}{c|}{ standard diagnosis } \\
\cline { 3 - 4 } \multicolumn{2}{c|}{} & Positive & Negative & \\
\hline \multirow{2}{*}{ CD-LFIA } & Positive & $\mathbf{8}$ & $\mathbf{4}$ & 12 \\
\cline { 2 - 4 } & Negative & $\mathbf{0}$ & $\mathbf{3 3}$ & 33 \\
\cline { 2 - 4 } & 8 & 37 & $\mathbf{4 5}$
\end{tabular}

$\begin{array}{ll}\text { Sensitivity } & 100 \%[63.1-100] \\ \text { Specificity } & 89.2 \%[74.6-97.0] \\ \text { NPV } & 100 \%[89.4-100] \\ \text { LR+ } & 9.3[3.7-23.3] \\ \text { LR- } & 0[0.0-0.91] \\ \text { Diagnostic Accuracy } & 91.1 \%[78.8-97.5]\end{array}$

Figure 2 Diagnostic performance of CD-LFIA [with 95\% Cl]. 
showed that the detection of IgG against DGP has a high specificity and a better sensitivity than IgG anti-tTG $[12-16,26]$ and this seems to be also true in case of IgA deficiency $[3,27,28]$.

A multi-analytic lateral-flow immunochromatographic assay (CD-LFIA) based on the rapid detection of both IgA and IgG anti-DGP and total IgA was previously shown to have a good diagnostic accuracy to rule out $\mathrm{CD}$ in high-risk paediatric and adult populations $[17,18]$. Since the test detects IgA and IgG antibodies against DGP and total IgA levels and it has a very good sensitivity and NPV value, it seems to us that the test could facilitate the management of paediatric patients, including undiagnosed IgAD, consulting in primary care centers for CD-related symptoms. Therefore, the aim of the present study was to evaluate its use as a tool to rule out CD in children suffering from IgAD.

Within the 45 paediatric IgAD patients included in the study, eight (8) were newly identified CDs, all of them were correctly identified by CD-LFIA. From the remaining 37 patients, which were considered as CD negative, 33 were correctly identified by CD-LFIA and four (4) were considered as false positive. Among them, one patient underwent duodenal biopsy and was subsequently diagnosed CD negative. A second patient (2.1 years old) had positive levels of IgG anti-DGP by ELISA. Although the recent ESPGHAN guidelines recommend the use of antibodies against DGP in children younger than 2, and several papers showed that antibodies against DGP outperform EMA and tTG antibodies in children under 2 years of age, the patient had no further follow-up and no endoscopic examination $[8,9,13,28,29]$. From the 33 CD negative patients, two (2) had a positive IgG antitTG serology but were diagnosed as CD negative based on their biopsy results and follow-up. Both patients were correctly diagnosed by CD-LFIA, and therefore CD-LFIA did not yield false negative results.

Limitations that apply to our study are foremost its retrospective nature in a high pretest population. The decision of conducting the study retrospectively in a secondary care setting was principally guided by the low prevalence of IgAD that could eventually increase the length of the study. Clinical symptoms were missing for ten (10) of the 45 patients included in this study. However, diagnostic agreement was found between the serologic IgA anti-tTG and CD-LFIA for all these patients, all being $\mathrm{CD}$ negatives.

\section{Conclusion}

With a sensitivity of $100 \%$ [63.1-100], a NPV of $100 \%$ [89.4-100] and a LR- of 0 [0.0-0.91], we showed here that CD-LFIA is a useful, non-invasive and rapid tool to rule out $\mathrm{CD}$ in primary care paediatric patients having CD-related symptoms and IgAD. By detecting in a single test, both IgA/IgG anti-DGP and total IgA, CD-LFIA appears to be well adapted to the recommendations concerning the systematic investigation of IgA levels in patients screened for $C D$ [8-10]. Patients having a positive CD-LFIA result could be then readily directed to secondary care setting for further evaluation by standard serology and biopsy.

\section{Competing interests}

CBD sits in the board of Augurix SA. SIA is an employee of Augurix SA. For the remaining authors there are no competing interests.

\section{Authors' contributions}

FB: conceptualized and designed the study, carried out the initial analysis and interpretation of the data, and participated to the writing of the manuscript. SIA: contributed to the data analysis and interpretation, and drafted the initial manuscript. CBD: conceptualized and designed the study, and participated to the writing of the manuscript. JG, LG, FR: performed data collection and analysis, and critically revised the manuscript. AL: contributed to study design and critically revised the manuscript. JB: conceptualized and designed the study and participated to the writing of the manuscript. All authors read and approved the final manuscript.

\section{Acknowledgements}

Simtomax ${ }^{\circledast}$ reagents were a generous gift from Augurix SA. No funding was secured for this study. The authors have indicated they have no financial relationship relevant to this article to disclose.

\section{Author details}

${ }^{1}$ Lyon-Sud Hospital-Immunology Laboratory, 69495, Pierre-Bénite Lyon, France. ${ }^{2}$ Augurix SA, Monthey $\mathrm{CH}-1870$, Switzerland. ${ }^{3}$ Hospices Civils de Lyon, Paediatric Hospital-Gastroenterology-Hepatology-Nutrition Department, Lyon 69677 Bron, France.

Received: 13 June 2014 Accepted: 15 October 2014

Published: 6 November 2014

\section{References}

1. Wang N, Hammarstrom L: IgA deficiency: what is new? Curr Opin Allergy Clin Immunol 2012, 12(6):602-608.

2. Jorgensen GH, Gardulf A, Sigurdsson MI, Sigurdardottir ST, Thorsteinsdottir I, Gudmundsson S, Hammarstrom L, Ludviksson BR: Clinical symptoms in adults with selective IgA deficiency: a case-control study. J Clin Immunol 2013, 33(4):742-747.

3. Villalta D, Alessio MG, Tampoia M, Tonutti E, Brusca I, Bagnasco M, Pesce G, Bizzaro N: Diagnostic accuracy of IgA anti-tissue transglutaminase antibody assays in celiac disease patients with selective IgA deficiency. Ann N Y Acad Sci 2007, 1109:212-220.

4. Cataldo F, Marino V, Ventura A, Bottaro G, Corazza GR: Prevalence and clinical features of selective immunoglobulin A deficiency in coeliac disease: an Italian multicentre study. Italian Society of Paediatric Gastroenterology and Hepatology (SIGEP) and "Club del Tenue" Working Groups on Coeliac Disease. Gut 1998, 42(3):362-365.

5. Chow MA, Lebwohl B, Reilly NR, Green PH: Immunoglobulin A deficiency in celiac disease. J Clin Gastroenterol 2012, 46(10):850-854.

6. Sanders DS, Patel D, Stephenson TJ, Ward AM, McCloskey EV, Hadjivassiliou M, Lobo AJ: A primary care cross-sectional study of undiagnosed adult coeliac disease. Eur J Gastroenterol Hepatol 2003, 15(4):407-413.

7. Anderson RP, Henry MJ, Taylor R, Duncan EL, Danoy P, Costa MJ, Addison K, Tye-Din JA, Kotowicz MA, Knight RE, Pollock W, Nicholson GC, Toh BH, Brown MA, Pasco JA: A novel serogenetic approach determines the community prevalence of celiac disease and informs improved diagnostic pathways. BMC Med 2013, 11(1):188.

8. Husby $S$, Koletzko S, Korponay-Szabo IR, Mearin ML, Phillips A, Shamir R, Troncone R, Giersiepen K, Branski D, Catassi C, Legeman M, Maki M, Ribes-Koninckx C, Ventura A, Zimmer KP: European Society for Pediatric Gastroenterology, Hepatology, and Nutrition guidelines for the diagnosis of coeliac disease. J Pediatr Gastroenterol Nutr 2012, 54(1):136-160. 
9. Lagerqvist $C$, Dahlbom I, Hansson T, Jidell E, Juto P, Olcen $P$, Stenlund $H$, Hernell O, Ivarsson A: Antigliadin immunoglobulin A best in finding celiac disease in children younger than 18 months of age. J Pediatr Gastroenterol Nutr 2008, 47(4):428-435.

10. Rubio-Tapia A, Hill ID, Kelly CP, Calderwood AH, Murray JA: ACG clinical guidelines: diagnosis and management of celiac disease. Am J Gastroenterol 2013, 108(5):656-676. quiz 677.

11. McGowan KE, Lyon ME, Butzner JD: Celiac disease and IgA deficiency: complications of serological testing approaches encountered in the clinic. Clin Chem 2008, 54(7):1203-1209.

12. Schwertz E, Kahlenberg F, Sack U, Richter T, Stern M, Conrad K, Zimmer KP, Mothes T: Serologic assay based on gliadin-related nonapeptides as a highly sensitive and specific diagnostic aid in celiac disease. Clin Chem 2004, 50(12):2370-2375.

13. Liu E, Li M, Emery L, Taki I, Barriga K, Tiberti C, Eisenbarth GS, Rewers MJ, Hoffenberg EJ: Natural history of antibodies to deamidated gliadin peptides and transglutaminase in early childhood celiac disease. J Pediatr Gastroenterol Nutr 2007, 45(3):293-300.

14. Niveloni S, Sugai E, Cabanne A, Vazquez H, Argonz J, Smecuol E, Moreno ML, Nachman F, Mazure R, Kogan Z, Gomez JC, Maurino E, Bai JC: Antibodies against synthetic deamidated gliadin peptides as predictors of celiac disease: prospective assessment in an adult population with a high pretest probability of disease. Clin Chem 2007, 53(12):2186-2192.

15. Dahle C, Hagman A, Ignatova S, Strom M: Antibodies against deamidated gliadin peptides identify adult coeliac disease patients negative for antibodies against endomysium and tissue transglutaminase. Aliment Pharmacol Ther 2010, 32(2):254-260.

16. Vermeersch P, Geboes K, Marien G, Hoffman I, Hiele M, Bossuyt X Serological diagnosis of celiac disease: comparative analysis of different strategies. Clin Chim Acta 2012, 413(21-22):1761-1767.

17. Bienvenu F, Besson Duvanel C, Seignovert C, Rouzaire P, Lachaux A, Bienvenu $\mathrm{J}$ : Evaluation of a point-of-care test based on deamidated gliadin peptides for celiac disease screening in a large pediatric population. Eur $J$ Gastroenterol Hepatol 2012, 24(12):1418-1423.

18. Benkebil F, Combescure C, Anghel SI, Besson Duvanel C, Schappi MG: Diagnostic accuracy of a new point-of-care screening assay for celiac disease. World J Gastroenterol 2013, 19(31):5111-5117.

19. Oberhuber G, Granditsch G, Vogelsang H: The histopathology of coeliac disease: time for a standardized report scheme for pathologists. Eur $J$ Gastroenterol Hepatol 1999, 11(10):1185-1194.

20. Maki M, Mustalahti K, Kokkonen J, Kulmala P, Haapalahti M, Karttunen T, Ilonen J, Laurila K, Dahlbom I, Hansson T, Hopfl P, Knip M: Prevalence of Celiac disease among children in Finland. N Engl J Med 2003, 348(25):2517-2524.

21. West J, Logan RF, Card TR, Smith C, Hubbard R: Fracture risk in people with celiac disease: a population-based cohort study. Gastroenterology 2003, 125(2):429-436.

22. Korponay-Szabo IR, Raivio T, Laurila K, Opre J, Kiraly R, Kovacs JB, Kaukinen K, Fesus L, Maki M: Coeliac disease case finding and diet monitoring by point-of-care testing. Aliment Pharmacol Ther 2005, 22(8):729-737.

23. Nemec G, Ventura A, Stefano M, Di Leo G, Baldas V, Tommasini A, Ferrara F, Taddio A, Citta A, Sblattero D, Marzari R, Tarcisio N: Looking for celiac disease: diagnostic accuracy of two rapid commercial assays. Am J Gastroenterol 2006, 101(7):1597-1600.

24. Nenna R, Tiberti C, Petrarca L, Lucantoni F, Mennini M, Luparia RP, Panimolle F, Mastrogiorgio G, Pietropaoli N, Magliocca FM, Bonamico M: The celiac iceberg: characterization of the disease in primary schoolchildren. J Pediatr Gastroenterol Nutr 2013, 56(4):416-421.

25. Alessio MG, Tonutti E, Brusca I, Radice A, Licini L, Sonzogni A, Florena A, Schiaffino E, Marus W, Sulfaro S, Villalta D: Correlation between IgA tissue transglutaminase antibody ratio and histological finding in celiac disease. J Pediatr Gastroenterol Nutr 2012, 55(1):44-49.

26. Prause C, Richter T, Koletzko S, Uhlig HH, Hauer AC, Stern M, Zimmer KP, Laass MW, Probst C, Schlumberger W, Komorowski L, Lieske R, Richter T, Hauer AC, Stern M, Uhlig HH, Laass MW, Zimmer KP, Mothes T: New developments in serodiagnosis of childhood celiac disease: assay of antibodies against deamidated gliadin. Ann N Y Acad Sci 2009, 1173:28-35.

27. Agardh D: Antibodies against synthetic deamidated gliadin peptides and tissue transglutaminase for the identification of childhood celiac disease. Clin Gastroenterol Hepatol 2007, 5(11):1276-1281.
28. Basso D, Guariso G, Fogar P, Meneghel A, Zambon CF, Navaglia F, Greco E, Schiavon S, Rugge M, Plebani M: Antibodies against synthetic deamidated gliadin peptides for celiac disease diagnosis and follow-up in children. Clin Chem 2009, 55(1):150-157.

29. Mubarak A, Gmelig-Meyling FH, Wolters VM, Ten Kate FJ, Houwen RH: Immunoglobulin $\mathrm{G}$ antibodies against deamidated-gliadin-peptides outperform anti-endomysium and tissue transglutaminase antibodies in children $<2$ years age. APMIS 2011, 119(12):894-900

doi:10.1186/1471-230X-14-186

Cite this article as: Bienvenu et al: Early diagnosis of celiac disease in IgA deficient children: contribution of a point-of-care test. BMC Gastroenterology 2014 14:186.

\section{Submit your next manuscript to BioMed Central and take full advantage of:}

- Convenient online submission

- Thorough peer review

- No space constraints or color figure charges

- Immediate publication on acceptance

- Inclusion in PubMed, CAS, Scopus and Google Scholar

- Research which is freely available for redistribution 